\section{ECMELLA: Uso combinado de dispositivos de asistencia ventricular izquierda en el manejo del shock cardiogénico postinfarto agudo al miocardio}

\author{
RENÉ HAMEAU D. ${ }^{1}$, FRANCESCO SORIANO ${ }^{2}$, JACOPO OREGLIA ${ }^{2}$, \\ RODRIGO MUÑOZ ${ }^{3}$, JOSÉ LUIS WINTER ${ }^{3}$, MARTÍN VALDEBENITO ${ }^{3}$, \\ JORGE QUITRAL ${ }^{1}$, DANTE LINDEFJELD ${ }^{3,4}$, NICOLAS VEAS ${ }^{3,4}$
}

\section{Combined use of left ventricular assist devices in cardiogenic shock. Report of one case}

We report a 55-year-old woman with a history of hypothyroidism and type 2 diabetes mellitus who consulted at the emergency room because of intermittent oppressive chest discomfort. At admission, electrocardiogram showed a complete atrioventricular block. A transthoracic echocardiogram disclosed severe left ventricular dysfunction. The patient developed cardiogenic shock that required the installation of the Impella system. An emergency coronary angiography showed an ostial occlusion of the anterior descending artery. Despite successful primary angioplasty, she persisted with refractory shock and progressive hypoxemia. A concomitant connection to the extracorporeal membrane oxygenation system (ECMO) was decided. The support of both devices allowed the stabilization of the patient and the improvement of perfusion parameters.

(Rev Med Chile 2020; 148: 409-413)

Key words: Heart-Assist Devices; Shock, Cardiogenic; Ventricular Dysfunction, Left.

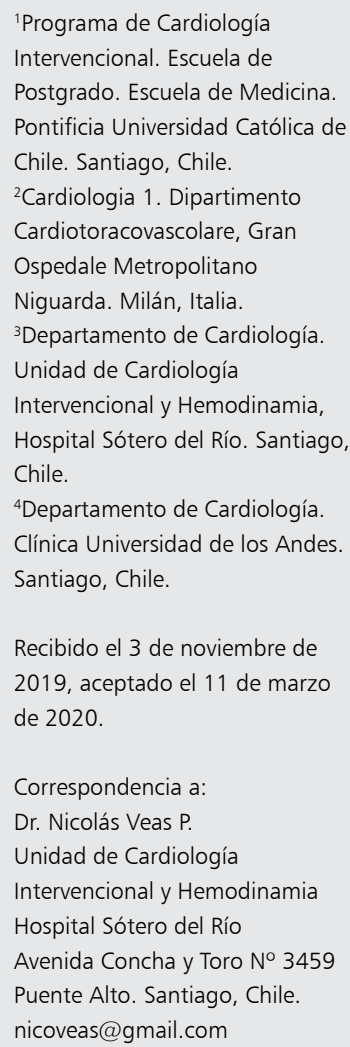

Recibido el 3 de noviembre de 2019, aceptado el 11 de marzo de 2020.

Correspondencia a:

Dr. Nicolás Veas $\mathrm{P}$.

Unidad de Cardiología Intervencional y Hemodinamia Hospital Sótero del Río Avenida Concha y Toro No 3459 Puente Alto. Santiago, Chile. nicoveas@gmail.com

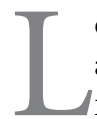

os pacientes que se presentan con un infarto agudo al miocardio (IAM) y shock cardiogé$\checkmark$ nico (SC) constituyen un grupo de muy alta mortalidad y la revascularización precoz es la principal medida de rescate. Aquellos que no logran estabilizarse precozmente, debieran considerarse en un protocolo de asistencia circulatoria antes de que se inicie el espiral de eventos que llevan al shock refractario.

Actualmente, los dispositivos más comúnmente utilizados son el balón de contrapulsación intraaórtica (IABP), Impella ${ }^{\mathrm{TM}}$ (Abiomed Inc, Danvers, MA, USA), TandemHeart ${ }^{\mathrm{TM}}$ (Cardiac Assist Inc., Pittsburgh, PA, USA) y el sistema de oxigenación por membrana extracorpórea (ECMO).

Nuestro país no es ajeno a esta realidad, contando con amplia experiencia en el uso del IABP $y$, recientemente, con una mayor disponibilidad de ECMO en centros de referencia regionales. Presentamos el caso de una paciente con shock cardiogénico tratado con el uso combinado de ECMO y del sistema Impella ${ }^{\mathrm{TM}}$ (ECMELLA)(Figura 1).

\section{Caso clínico}

Paciente de 55 años, sexo femenino, con antecedentes de diabetes mellitus tipo 2 e hipo- 


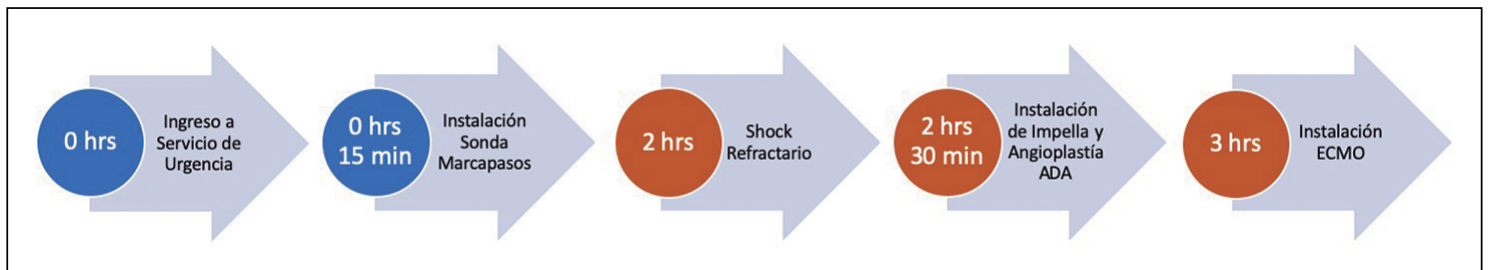

Figura 1. Línea temporal de las intervenciones terapéuticas. ADA: Arteria descendente anterior, ECMO: Oxigenación por membrana extracorpórea.

tiroidismo que consulta por cuadro de $3 \mathrm{~h}$ de dolor torácico opresivo subintrante. Al examen físico destacaba una paciente mal perfundida, con compromiso sensorial y ventilatorio progresivo.

El electrocardiograma de ingreso mostró un bloqueo AV completo, por lo que se instaló una sonda marcapasos transitoria y el ecocardiograma transtorácico evidenció una disfunción ventricular izquierda (VI) grave (FEVI 20-25\%) con hipokinesia difusa.

A pesar del apoyo con ventilación mecánica invasiva y drogas vasoactivas, se constató hipoxemia y acidosis láctica progresivas en el contexto de falla multiorgánica. Los exámenes de laboratorio mostraron: Troponinas $11.000 \mathrm{pg} / \mathrm{mL}$, hematocrito $40 \%$, creatinkinasa total /MB: 5.400/ 235 $\mathrm{U} / \mathrm{L}$, creatininemia $2,2 \mathrm{mg} / \mathrm{dL}$ y ácido láctico 12 $\mathrm{mmol} / \mathrm{L}$.

Se decidió la instalación del sistema Impella $\mathrm{CP}^{\mathrm{TM}}$ (Abiomed Inc, Danvers, MA, USA) vía arteria femoral (AF) izquierda $14 \mathrm{~F}$ y estudio coronario vía $\mathrm{AF}$ derecha de urgencia. Se evidenció una oclusión de arteria descendente anterior ostial, realizándose angioplastía con stent liberador de everolimus guiado por ultrasonido intracoronario (IVUS) (Figura 2). A pesar de lo anterior, persistía hipoperfundida con un VI dilatado (diámetro telesistólico [DTS] $45 \mathrm{~mm}$ ), capilar pulmonar persistentemente alto (PCP $>26 \mathrm{mmHg}$ ), clearance de lactato $<10 \%$ en $2 \mathrm{~h}$, así como hipoxia refractaria. La paciente fue presentada al shock team, formado en el Hospital Sótero del Río, siguiendo la tendencia de la Cardiología Intervencional, en que un equipo de especialistas que incluye a cardiólogo intervencionista, cirujano cardiaco, especialista en insuficiencia cardiaca y en cuidados críticos cardiológicos, analizan y definen una conducta, se decidió mantener con Impella e implantar ECMO veno-arterial (ECMO VA) como medida

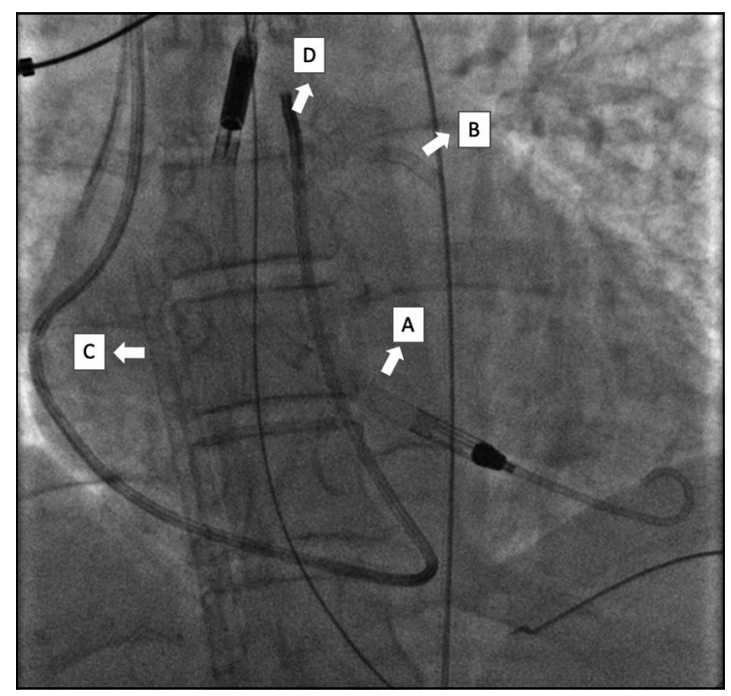

Figura 2. Imagen fluoroscópica postinstalación de asistencia ventricular. A) Dispositivo Impella (P; B) Stent liberador de Everolimus en arteria descendente anterior; C) Cánula venosa de ECMO; D) Catéter de Swan Ganz.

de rescate para mejorar la oxigención y perfusión sistémicas con una cánula $21 \mathrm{~F}$ venosa femoral derecha y una cánula 19F AF derecha (ECMELLA). El razonamiento inicial fue mantener el Impella para favorecer la descompresión VI, disminución de la PCP y la recuperación del miocardio atontado.

La paciente evolucionó con recuperación parcial de la función ventricular (FEVI 28-30\%), de la dilatación ventricular (DTS $40 \mathrm{~mm}$ ) y la PCP $20 \mathrm{mmHg}$. La normalización progresiva de los parámetros perfusionales permitió el retiro de Impella a las $48 \mathrm{~h}$ y ECMO VA a las $72 \mathrm{~h}$. Tras 10 días de hospitalización, se indicó el alta en buenas condiciones generales, pero con persistencia de una disfunción VI grave, actualmente se mantiene en estudio de pretrasplante cardiaco. 


\section{Discusión}

Presentamos un caso del uso combinado de 2 dispositivos de asistencia VI en el contexto de un shock cardiogénico post IAM. El shock cardiogénico (SC) constituye un estado crítico de hipoperfusión de órgano blanco por disfunción cardíaca primaria ${ }^{1}$ con una incidencia de $8 \%$ a $12 \%$ en el IAM con supradesnivel de ST (SDST), $5 \%$ en el IAM sin SDST y una mortalidad entre $40 \%$ y $50 \%^{2}$. En el registro SHOCK, la causa predominante fue la falla izquierda $(78,5 \%)$, seguido de la insuficiencia mitral severa $(6,9 \%)$, rotura del septum IV $(3,9 \%)$, falla derecha $(2,8 \%)$ y taponamiento cardiaco $(1,4 \%)^{3}$.

Sepúlveda et cols. ${ }^{4}$ analizaron los datos nacionales del registro GEMI entre los años 2001 y 2009 e incluyó a 7.548 pacientes ingresados en Killip IV secundario a un IAM. La incidencia de SC fue de $3,7 \%$ y la mortalidad global de $55 \%$. Aquellos centros con angioplastía de urgencia tuvieron una mortalidad significativamente menor $(49,0 \%$ vs 62,2\%; p 0,028).

Por su parte, los dispositivos de asistencia circulatoria surgen como una línea terapéutica prometedora y actualmente cuentan con una recomendación IIb para uso en shock refractario ${ }^{5}$.

Shah et cols. analizaron un registro con 144.254 casos de SC entre 2005 y 2014, de los cuales, $55,4 \%$ eran asociados a un IAM. Se observó una disminución del uso de IABP $(29,8 \rightarrow 17,7 \%)$ $\mathrm{y}$ un incremento discreto en la utilización tanto de Impella $(0,1 \rightarrow 2,6 \%)$ como de ECMO $(0,3 \rightarrow$ $1,3 \%)$. El grupo etáreo en que más se utilizó la asistencia circulatoria fue entre 51 y 65 años (32,3\%).

Impella $^{\mathrm{TM}}$ es un dispositivo de flujo axial no-pulsátil, con un rotor de aproximadamente $50.000 \mathrm{rpm}$, que provee de flujo anterógrado, además de permitir una descarga del VI, disminuir la PCP y del consumo de $\mathrm{O}_{2}$ miocárdico. En Estados Unidos de Norteamérica fue aprobado en 2008 y cuenta con 3 versiones: Impella $2.5^{\mathrm{TM}}$, que provee un flujo máximo de $2,5 \mathrm{~L} / \mathrm{min}$ para un soporte hemodinámico parcial; Impella $\mathrm{CP}^{\mathrm{TM}}$ que aporta entre 3 y $4 \mathrm{~L} / \mathrm{min}$ y el Impella $5.0^{\mathrm{TM}}$ con un flujo de hasta $5,0 \mathrm{~L} / \mathrm{min}$.

Dentro de sus ventajas están la necesidad únicamente de acceso arterial, reduciendo el tiempo de inserción hasta cerca de 10 minutos en centros con experiencia.

El ISAR-SHOCK fue un estudio aleatorizado que evaluó Impella $2.5^{\mathrm{TM}}(\mathrm{n}=12)$ en pacientes con SC vs un IABP $(n=13)$. El objetivo primario fue el cambio en el índice cardiaco 30 min después del implante del dispositivo. Todos los pacientes recibieron el Impella posterior a la angioplastía. El incremento fue de $0,491 / \mathrm{min} / \mathrm{m}^{2}$ con Impella ${ }^{\mathrm{TM}}$ vs $0,11 \mathrm{l} / \mathrm{min} / \mathrm{m}^{2}$ con IABP (p 0,02 ), observándose también mejorías significativas respecto de la PAm y una mayor reducción en los niveles de lactato. La mortalidad a 30 días fue de $46 \%$ en ambos grupos ${ }^{6}$.

El estudio IMPRESS comparó Impella CP $(\mathrm{n}=24)$ vs IABP $(\mathrm{n}=24)$ en pacientes con SC como complicación de un IAM (años 2012-2015). El Impella ${ }^{\mathrm{TM}}$ fue insertado previo a la angioplastía en $21 \%$ de los pacientes. La mortalidad por cualquier causa con IABP o Impella ${ }^{\mathrm{TM}}$ fue similar tanto a 30 días $(50 \%$ y $46 \%$, respectivamente; $\mathrm{p}=0,92)$ como a los 6 meses $(50 \%$ en ambos grupos; $\mathrm{p} 0,923)^{7}$.

El subanálisis del estudio PROTECT II sugiere que los beneficios de Impella ${ }^{\mathrm{TM}}$ pudieran provenir del hecho de permitir una revascularización más completa, así como de una reducción en las tasas de falla renal a 30 y 90 días $^{8}$.

$\mathrm{Al}$ proveer tanto soporte respiratorio como cardiaco, el ECMO VA constituye otro de los dispositivos de primera línea. Sus potenciales ventajas son una rápida instalación por vía periférica, aplicabilidad en casos de arritmias malignas y mejoría rápida de la oxigenación ${ }^{9}$. ECMO puede proveer un soporte completo con flujos hasta $3-4 \mathrm{~L} / \mathrm{min}$ en aquellos de inserción periférica y $5 \mathrm{~L} / \mathrm{min}$ en aquellos centrales.

En un metaanálisis de 13 estudios incluyendo a pacientes con paro cardiaco refractario $(\mathrm{n}=3.098)$ o SC postinfarto $(n=235)$, Ouweneel et cols. ${ }^{10}$ describen que el uso de ECMO VA obtuvo una sobrevida a 30 días que fue $33 \%$ superior a la observada con IABP (IC 95\% 14\%-52\%; p < 0,001; NNT 13), pero sin diferencias al compararse con TandemHeart ${ }^{\mathrm{TM}}$ o Impella $^{\mathrm{TM}}$ (IC 95\% -21 a 14\%; p 0,70; NNH 33).

Dentro de las limitaciones se encuentra el riesgo de isquemia de extremidades y eventos tromboembólicos, así como el aumento de la postcarga VI, que podría dificultar la recuperación cardiaca o favorecer el edema pulmonar ${ }^{11}$. También en ventrículos previamente dilatados pudiera favorecer la formación de trombos apicales ${ }^{12}$.

Por esto, muchos autores han planteado el 
uso concomitante de dispositivos, especialmente para ayudar a descargar el VI, disminuyendo la PCP y desplazando la curva presión-volumen del VI hacia valores más bajos ${ }^{13}$. La asociación también tendría utilidad en situaciones con marcada hipoxemia o estados de hipoperfusión y falla multisistémica establecida en quienes la simple mantención del flujo anterógrado no basta para revertir este proceso.

Si bien no existen estudios aleatorizados, existe evidencia preliminar que sugiere un beneficio. Un metaanálisis de 29 estudios, realizado por Li et cols, sugirió que la combinación de ECMO VA + IABP logró reducir en 10\% la mortalidad intrahospitalaria, sin aumentar la incidencia de complicaciones neurológicas ni vasculares ${ }^{14}$.

Respecto a la estrategia "ECMELLA", Schrage et al. ${ }^{15}$ reportan una serie de 106 pacientes cursando con SC ( $60 \%$ por síndrome coronario agudo) y tratados con esta estrategia. A 30 días, la sobrevida fue de $35,8 \%$, siendo significativamente superior a la calculada por los score SAVE (20\%) y SAPS II (6,9\%). En otra serie de 157 pacientes, Pappalardo et cols. reportaron una reducción significativa de la mortalidad de la combinación ECMO + Impella vs solo ECMO $(47 \% \text { vs } 80 \%, \mathrm{p}<0,001)^{16}$.

Russo et cols. ${ }^{17}$ analizaron 17 estudios observacionales con 3.997 pacientes con SC (40,9\% de causa isquémica) y compararon aquellos que recibieron un dispositivo de asistencia (Impella, IABP, etc.) adicional al ECMO VA ( $\mathrm{n}=1.696$; $42 \%$ ) vs aquellos que no lo hicieron. La mortalidad fue significativamente menor en aquellos que recibieron algún dispositivo de descarga (RR 0,79; IC 95\% 0,72-0,87; $\mathrm{p}<0,00001)$. Este grupo presentó mayor incidencia de hemólisis (RR 2,15; 95\% CI: $1,49$ a 3,$11 ; p<0,0001)$, sin otras diferencias en los objetivos secundarios.

En conclusión, la alta mortalidad del shock cardiogénico nos obliga a continuar explorando nuevas estrategias y el uso combinado de dispositivos de asistencia VI pudiera ser una alternativa que contribuya a mejorar estos resultados clínicos.

\section{Referencias}

1. Van Diepen S, Katz JN, Albert NM, Henry TD, Jacobs AK, Kapur NK. Contemporary Management of Cardiogenic Shock: A Scientific Statement from the
American Heart Association. Circulation 2017; 136 (16): 232-68.

2. Kolte D, Khera S, Aronow WS, Mujib M, Palaniswamy C, Sule S. Trends in incidence, management, and outcomes of cardiogenic shock complicating ST-elevation myocardial infarction in the United States. J Am Coll Cardiol 2016; 117 (1): 1-9.

3. Hochman JS, Sleeper LA, Godfrey E, McKinlay SM, Sanborn T, Jacques C. SHould we emergently revascularize occluded coronaries for cardiogenic ShocK: An international randomized trial of emergency PTCA/ CABG-trial design. Am Hear J 1999; 137 (2): 313-21.

4. Sepúlveda P, Nazzal C, Soto G, Prieto JC, Sanhueza P, Campos $\mathrm{P}$, et al. Características clínicas y tratamiento de pacientes con infarto agudo del miocardio con supradesnivel st y shock cardiogénico: 9 años del Registro GEMI. Rev Med Chile 2012; 31: 194-201.

5. Ibáñez B, James S, Agewall S, Antunes MJ, Bucciarelli-Ducci C, Bueno H, et al. 2017 ESC Guidelines for the management of acute myocardial infarction in patients presenting with ST-segment elevation. Eur Heart J 2017; 39 (2): 119-77.

6. Seyfarth M, Sibbing D, Bauer I, Fröhlich G, Bott-Flügel L, Byrne R, et al. A Randomized Clinical Trial to Evaluate the Safety and Efficacy of a Percutaneous Left Ventricular Assist Device Versus Intra-Aortic Balloon Pumping for Treatment of Cardiogenic Shock Caused by Myocardial Infarction. J Am Coll Cardiol 2008; 52 (19): 1584-8.

7. Ouweneel DM, Eriksen E, Sjauw KD, van Dongen IM, Hirsch A, Packer E, et al. Percutaneous Mechanical Circulatory Support Versus Intra-Aortic Balloon Pump in Cardiogenic Shock After Acute Myocardial Infarction: IMPRESS trial. J Am Coll Cardiol 2017; 69 (3): 278-87.

8. O'Neill WW, Kleiman NS, Moses J, Henriques JPS, Dixon S, Massaro J, et al. A prospective, randomized clinical trial of hemodynamic support with impella 2.5 versus intra-aortic balloon pump in patients undergoing high-risk percutaneous coronary intervention: The PROTECT II study. Circulation 2012; 126 (14): 1717-27.

9. Abrams D, Garan AR, Abdelbary A, Bacchetta M, Bartlett RH, Beck J, et al. Position paper for the organization of ECMO programs for cardiac failure in adults. Intensive Care Med 2018; 44 (6): 717-29.

10. Ouweneel DM, Schotborgh JV, Limpens J, Sjauw KD, Engström AE, Lagrand W, et al. Extracorporeal life support during cardiac arrest and cardiogenic shock: a systematic review and meta-analysis. Intensive Care Med 2016; 42 (12): 1922-34.

11. Aissaoui N, Caudron J, Leprince P, Fagon JY, Lebreton $\mathrm{G}$, Combes A, et al. Right-left ventricular interdepen- 
dence: a promising predictor of successful extracorporeal membrane oxygenation (ECMO) weaning after assistance for refractory cardiogenic shock. Intensive Care Med 2017; 43 (4): 592-4.

12. Hireche-Chikaoui H, Grübler MR, Bloch A, Windecker S, Bloechlinger S, Hunziker L. Nonejecting hearts on femoral veno-arterial extracorporeal membrane oxygenation: Aortic root blood stasis and thrombus formation-A case series and review of the literature. Crit Care Med 2018; 46 (5): e459-e464.

13. Li Y, Yan S, Cai L, Qiaoni Z. Does VA-ECMO Plus Impella Work in Refractory Cardiogenic Shock? JACC Hear Fail. 2019; 7 (4): 364.

14. Li Y, Yan S, Gao S, Liu M, Lou S, Liu G, et al. Effect of an intra-aortic balloon pump with venoarterial extracorporeal membrane oxygenation on mortality of patients with cardiogenic shock: A systematic review and meta-analysis. Eur J Cardiothorac Surg 2019; 55 (3): 395-404.

15. Schrage B, Burkhoff D, Rübsamen N, Becher PM, Schwarzl M, Bernhardt A, et al. Unloading of the Left Ventricle During Venoarterial Extracorporeal Membrane Oxygenation Therapy in Cardiogenic Shock. JACC Hear Fail 2018; 6 (12): 1035-43.

16. Pappalardo F, Schulte C, Pieri M, Schrage B, Contri R, Soeffker G, et al. Concomitant implantation of Impella ${ }^{\circledR}$ on top of veno-arterial extracorporeal membrane oxygenation may improve survival of patients with cardiogenic shock. Eur J Heart Fail 2017; 19 (3): 40412.

17. Russo J, Aleksova N, Pitcher I, Couture E. Left Ventricular Unloading During Extracorporeal Membrane Oxygenation in Patients With Cardiogenic Shock. J Am Coll Cardiol 2019; 73 (6): 654-62. 